\title{
Applying Graphical Design Techniques to Graph Visualisation
}

\author{
Martyn Taylor, Peter Rodgers \\ University of Kent, University of Kent \\ \{mt63@kent.ac.uk, P.J.Rodgers@kent.ac.uk\}
}

\begin{abstract}
This paper contains details of new criteria for graph layout, based on the concepts used in graphical design. Current graph layout criteria have been shown to be effective in measuring the quality of a graph layout, but they are ad-hoc and often miss subtle appearance considerations such as balance and distribution.

We discuss how the principles concerning the layout of text and diagrams from design can be applied to graph layout and show how two new metrics can be implemented based on these criteria. We also give preliminary examples of layout generated using the new metrics.
\end{abstract}

\section{Introduction}

Over the past few years there has been much work in the field of graph visualisation with the goal of improve the layout of graphs to increase users' comprehension of the information represented by the diagram. A widely applied technique in this field is to measure the quality of a layout using a number metrics. The result of these can then be combined to give an overall fitness for the graph drawing. At present, commonly applied metrics are based on software designers' perceptions of what constitutes a good graph drawing, and as such they do not always correspond to users' perception of what a good layout is.

In this paper we attempt to improve this work by examining the criteria used by graphical designers in producing a pleasing and comprehensible layout for diagrams and text. The results of our work are new metrics based on graphical design techniques that may be combined with existing metrics to give a more accurate measurement of the quality of the graph. We also give some examples of preliminary work in using these metrics to lay out graphs using a multi-criteria optimiser.

There are many existing metrics to measure the aesthetics or usability of a graph, listed in Section 2.1. However, little use of the parallel work in the field of graphical design as been applied to graph visualization. There are also many conventions used when laying out a diagram or a page, many of these are listed in Section
2.2. In both fields there has been shown to be a positive correlation between the criteria when applied to displayed material and usability [2] [7] [8].

In order to adapt graphic design criteria for use in graph layout, it is necessary to develop measurement methods for them. We illustrate this with metrics that attempt to measure two criteria, Concentration and Homogeneity. Concentration is how evenly distributed a graph is within the area it occupies. Homogeneity is how even the distribution of graph elements is between top and bottom, or left and right.

The rest of the paper is organised as follows. The largest section, Section 3 describes implementations for both the more widely used graph drawing criteria and two criteria, Concentration and Homogeneity, which have been used in graphic design. Section 3.3 illustrates the use of the criteria in a multi-criteria graph drawing system. Section 4 gives our conclusions and details some further work to move on from this preliminary investigation.

\section{Design Criteria}

In this section we consider a number of criteria for producing good layout. First, we briefly summarise the commonly adopted graph drawing criteria. We then look at criteria commonly used in the field of graphic design and discuss how they might be used in a graph context.

\subsection{Graph Drawing Criteria}

When considering the graph drawing metrics in isolation, the commonly adopted aesthetics [2] [4] include:

Edge crossings are generally considered to be one of the major factors in reducing understanding of a graph. Empirical studies support this intuitive notion [7].

Total Edge Bends: A human eye can follow a straight edge more easily than an edge that zigzags through the picture. Reducing this simplifies the graph in terms of visualisation and for certain applications (such as circuit diagrams where bends in wires are trouble spots) can be detrimental to the overall layout.

Uniform Bends: Restrictions can be placed on the angles and positions of bends in lines to make the diagram more regular. 
Area: Visual space is often at a premium and reducing the overall area of the bounding box of the graph is often desirable. The shape of the graph can also be important, so that it has a certain aspect ratio, hence fitting well on a page or screen.

Total Edge Length: Minimisation of the sum of the length of the edges should cause a reduction of the area of the graph. Minimising the maximum edge length can also be beneficial.

Uniform Edge Lengths often make the regions in a graph into regular shapes that should be easier to visualise than complex shapes and should give each edge the same visual emphasis.

Angular Resolution: A small angle between edges emanating from a node makes the edges difficult to distinguish.

Symmetry: It is often important to reflect a graph's symmetry in its visualisation, however this is not a trivial task. Symmetry can be local (a subgraph of the graph) or global (the whole graph).

Node Separation: Nodes should be sufficiently far apart from their nearest neighbour to be easily distinguished and to avoid occlusion.

Node Clustering: Displaying close relationships between nodes by placing related nodes closer together allows for good visualisation of the groups within a graph.

\subsection{Graphical Design Based Criteria}

These criteria are more generalised than the graph drawing criteria and have been drawn from the principles underlying the layout of graphics, text and user interface components. Many of the criteria overlap with those relating to graph drawing.

Ngo, Teo and Byrne [6] have collated a series of fourteen metrics that can be applied to a layout to measure its aesthetic appeal. These metrics try to quantify different visual effects. However the ways they are calculated, and their relative weighting, have not been validated for their efficiency and accurate representation of human aesthetic measurement. The different metrics are described below.

Balance is the distribution of optical weight within the layout. Optical weight refers to the perception that some objects appear to be heavier than others. Dark colours, unusual shapes, and larger objects are heavier, whereas light colours, regular shapes, and small objects are lighter. Balance is achieved by providing an equal weight of visual elements in each quadrant.

Equilibrium is the stabilisation of the layout in the centre of the visual area. On a screen this involves making sure the overall centre of mass of the objects is the centre of the screen.

Symmetry is the extent to which the layout is symmetrical in three directions: vertically, horizontally, and diagonally.

Sequence refers to the arrangement of objects in a layout in a way that facilitates the movement of the eye through the information displayed. Normally the eye, trained by reading, starts from the upper left and moves back and forth across the display to the lower right. Perceptual psychologists have found that certain things attract the eye; it moves from big objects to small objects, from bright colours to subdued colours, from colour to black and white, and from irregular shapes to regular shapes.

Aspect Ratio (Cohesion): The term aspect ratio refers to the relationship of width to height. Changing the aspect ratio of a visual field may affect eye movement patterns sufficiently to account for some of the performance differences. The aspect ratio of a visual field should stay the same during the scanning of a display.

Proportion relates to the optimum aspect ratio, as well as the optimum size for visual elements, preferred by different people and cultures. Marcus [5] describes the following shapes as aesthetically pleasing: Square (1:1), Square root of two (1:1.414), Golden rectangle (1:1.618), Square root of three $(1: 1.732)$, Double square $(1: 2)$. Aesthetically pleasing proportions should be considered for major components in the layout.

Unity: Creating coherence, a totality of elements that is visually all one piece. With unity the elements seem to belong together, dovetailing so completely that they are seen as one thing. Using similar sizes, shapes, or colours for related information and leaving less space between elements of a screen than the space left at the margins achieves unity.

Simplicity is achieved is by optimising the number of elements on a screen and minimising the alignment points. Tullis [9] has derived a measure of screen complexity for text-based screens based on the work of Bonsiepe [3], who proposed a method of measuring the complexity of typographically designed pages through the application of information theory.

Density differs from the standard graph notion of density, which describes the relationship between the number of nodes and edges in a graph. In design, this is the extent to which the layout is covered with objects. Optimal density is achieved by minimising the density levels without making the layout too sparse. This result from minimising the number of visual elements being displayed or by making sure they are evenly spaced so the density is constant across the layout. A measure of density, derived by Tullis [9], is the percentage of character positions on the entire frame containing data.

Regularity is a uniformity of elements based on some principle or plan. Establishing consistently spaced horizontal and vertical alignment points for visual elements and minimising the number alignment points achieves regularity in screen design.

Economy is the careful and discreet use of display elements to get the message across as simply as possible. Economy is achieved by using as few styles, displays techniques and colours as possible.

Homogeneity: The relative degree of homogeneity of a composition is determined by how evenly the objects are distributed among the four quadrants of the 
layout. A more even distribution amongst the quadrants gives a more homogeneous graph.

Rhythm refers to regular patterns of changes in the elements. This use of order with variation helps to make the appearance exciting. Rhythm is accomplished through variation of arrangement, dimension, number and form of the elements.

Order (and conversely Complexity): The measure of order is the weighted sum of the above measures for a layout. The scale may be considered with order at one end and extreme complexity at the other.

Other suggested aesthetic qualities for layouts [1] are included below.

Hierarchy and Focus: An objects importance in a layout can be determined by its prominence of position.

Tension: Using heightened visual weight or close position, in moderation, creates tension between objects and can enhance the aesthetic quality of a layout. However over use makes a layout difficult to understand.

Depth: Manipulating the scale of objects and zorder can create an illusion of 3D position. The object with the largest apparent size will dominate the foreground of the layout.

Scale: As with depth, the relative size of elements affects the prominence of objects within the layout.

Movement Usually figurative, with elements angled or poised like bodies in motion, movement can also be created with such optical effects as linear repetition, visual vortexes and the like. Used deliberately, suggested movement can have a marked emotional and physical impact on a viewer.

\subsection{Design Criteria for Graph Drawing}

Some of the criteria described above are very similar. For example, symmetry and aspect ratio which both appear in graph and graphical design criteria. Aspect Ratio has the same meaning in both metrics, however Symmetry tends to be a global metric when looking at page layout in graphical design whereas few graphs will be globally symmetric and symmetry will tend to be localised in clusters. Some criteria seem related in a less direct way, for instance Economy of layout relates to several of the graph aesthetics, as adding both edge bends and edge crossings creates complexity and reduces Economy.

Candidates for new criteria for graph drawing must be both relevant to graph layout and measurable. The ability to use a quantifiable metric representing a particular criterion is important when measuring the overall goodness of a diagram. Many of the criteria above are too abstract to be applied in such a way; for instance, there has been little success in developing metrics for symmetry in graph for it to be applied as a global metric.

The following design criteria have been identified as potentially useful in graph drawing.

Homogeneity is a measure of effective use of area and equal density across the layout. A graph that is spread out equally should have nodes spaced across the canvas and thus have a homogenous number of nodes in each quadrant. A homogenous graph should be relatively well balanced and centred, so long as each element of the graph has equal visual weight, because the nodes will be equally distributed across the quadrants of the layout.

Density, which we rename to Concentration in order to differentiate it from the traditional graph meaning of density, measures a graph layouts' distribution of nodes. A layout that has an uneven distribution of nodes has a poor concentration, whereas a layout without dense groups of nodes and having an even spread of nodes will have a good concentration.

In the next section we describe in detail our metrics for measuring both Homogeneity and Concentration in a graph, and demonstrate how they can be applied in a multi-criteria optimising approach.

\section{Implementation of the Criteria}

The section below details the implementations of both the graphical design inspired criteria and the existing graph criteria as well as an evaluation of the preliminary results comparing graphs laid out using only the existing graph criteria compared to graphs laid out using both sets of criteria.

\subsection{Graphical Design Based Metrics}

Concentration measures if there is an equal spread of nodes throughout the layout. To simplify the calculations a $\lceil\sqrt{n}\rceil \times\lceil\sqrt{n}\rceil$ grid is used for an $n$ node graph and an optimal solution should have an even spread of nodes throughout the grid with either zero or one node in each grid area. The following formula is used to calculate concentration.

$$
\begin{array}{ll}
\forall x, y: & (x \in \mathbb{N}, 1 \leq x \leq\lceil\sqrt{n}\rceil, y \in \mathbb{N}, 1 \leq y \leq\lceil\sqrt{n}\rceil) \\
\operatorname{Grid}_{(x, y)} & =\text { Count of nodes within grid area } \\
M_{\text {Density }} & =\frac{\sum \operatorname{Max}\left(\operatorname{Grid}_{(x, y)}-1: 0\right)}{n-1}
\end{array}
$$

In the graphs below there are always nodes on the edge of the grid. This is because a bounding box around the graph is used to calculate the grid rather than a fixedsized grid as it allows the metric to cater for graph that exceed the bounds of the page and allows for later resizing of the graphs.

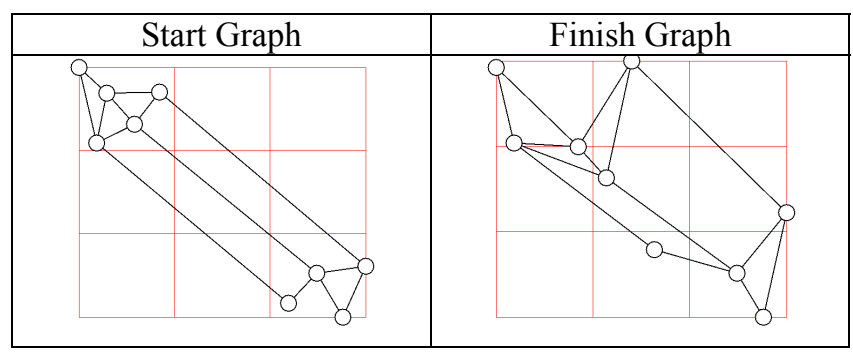

The graph above shows how applying only the concentration metric to a multi-criteria optimiser can be 
used to separate nodes. The nodes in each cluster have been forced apart and, although an optimal concentration has not been reached, there are now only one or two nodes in each grid.

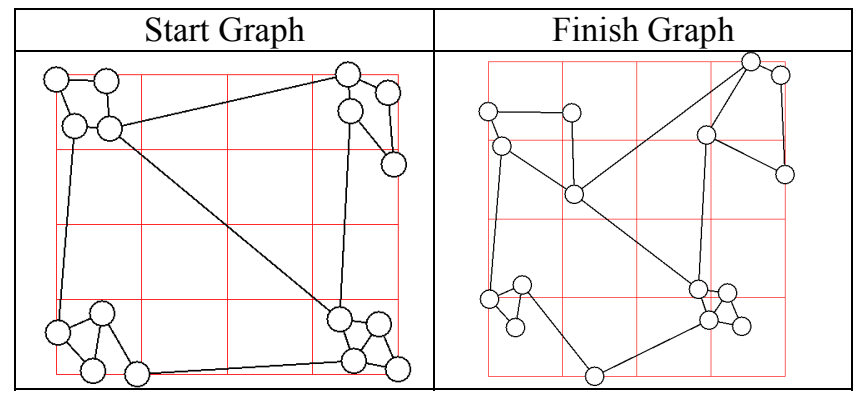

These graphs highlight a problem with the concentration metric when it is used on its own. The metric measures the number of nodes within each grid square but it does not measure their position. This means that there can be nodes on either side of the boundary between grid squares and the metric will register it as a good layout even though the node separation can be bad.

Homogeneity is a measure of how evenly the objects are distributed amongst the four quadrants of the display and is measured using a comparison between the combinations of ways objects can be organised for the given distribution compared to an optimal distribution.

Ngo, Teo and Byrne [6] define $W$ as the number of combinations of ways a group of $n$ objects can be arranged for a given distribution amongst the quadrants. Given $n$ objects, there are $n$ ! different ways of ordering them ( $n$ ways to pick the first object, $(n-1)$ ways to pick the second object, and so on). If the objects are split up between four quadrants, so there are $n_{\mathrm{UL}}, n_{\mathrm{UR}}, n_{\mathrm{LL}}$ and $n_{\mathrm{LR}}$ objects in the upper-left, upper-right, lower-left and lower-right quadrants respectively, then there are $n_{\mathrm{j}}$ ! ways of ordering the objects in quadrant $\mathrm{j}$. Therefore there are $n_{\mathrm{UL}} ! n_{\mathrm{UR}} ! n_{\mathrm{LL}} ! n_{\mathrm{LR}} !$ ways of ordering all objects in the four given quadrants. Given that the order of objects in the quadrants does not matter then $W$ is defined as

$$
W=\frac{n !}{n_{\mathrm{UL}} ! n_{\mathrm{UR}} ! n_{\mathrm{LL}} ! n_{\mathrm{LR}} !} .
$$

It follows that $W$ is maximum when the $n$ objects are evenly allocated to the quadrants. Therefore with $n$ objects each quadrant will contain $\lfloor n / 4\rfloor_{\text {objects. To ensure }}$ that $n / 4$ is an integer value, and that $W_{\max }$ is greater than or equal to $W$ for all values even when $n$ is not exactly divisible by $4, n / 4$ is rounded down. Therefore $W_{\max }$ is defined as:

$$
W_{\max }=\frac{n !}{(\lfloor n / 4\rfloor !)^{4}} .
$$
as:

Therefore the Homogeneity metric can be defined

$$
M_{\text {Homogeneity }}=1-\frac{W}{W_{\max }} .
$$

This gives a metric value between 0 (optimal distribution) and 1 (worst distribution).

Again, a bounding box around the graph is used to calculate the position of the quadrants and so some nodes will always appear on the outer bounds of the grid.

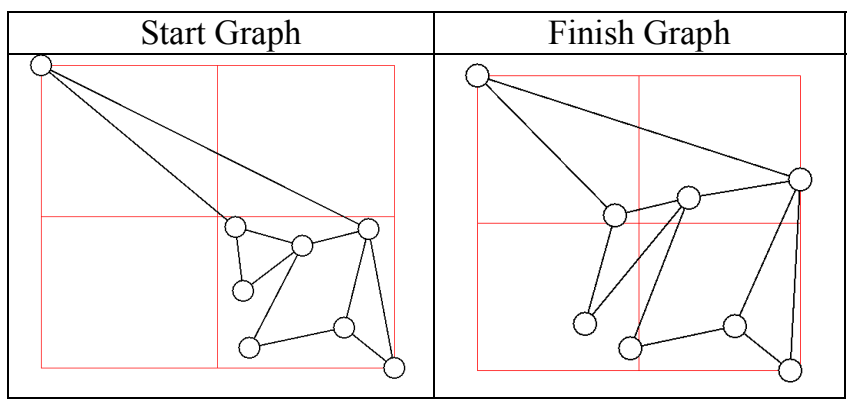

The graph above shows how the homogeneity metric moves the nodes so they are spread equally throughout the quadrants of the graph.

\begin{tabular}{|c|c|}
\hline Start Graph & Finish Graph \\
\hline
\end{tabular}

Similar to the density metric, the metric does not measure the position of nodes within the quadrant. As the graph above shows with little movement a node can move from one quadrant to another and improve the metric. The graphs also show that this factor can produce nodes with a small degree of separation with nodes on either side of the boundary between grids.

\subsection{Existing Graph Metrics}

In this section we briefly describe the standard graph drawing metrics implemented to allow comparison with the new design inspired metrics.

Angular Resolution is only calculated for edges smaller than the angular resolution, in this case $15^{\circ}$. The metric for each node is calculated using the square of the angle of incidence between edges at a node divided by the square of the angular resolution.

$\exists$ Edge $_{\mathrm{i}}$, Edge $_{\mathrm{j}}$ : where Edge $_{\mathrm{i}}$ and Edge $\mathrm{j}_{\mathrm{j}}$ connect to Node $_{\mathrm{n}}$ $\theta=$ Angle between Edge $_{\mathrm{i}}$ and Edge ${ }_{\mathrm{j}}$

$$
\begin{aligned}
& \operatorname{Metric}_{i, j}=1-\frac{\operatorname{Min}(\theta, 15)^{2}}{15^{2}} \\
& \text { Metric }_{\text {Angular }}=\sum \text { Metric }_{i, j}
\end{aligned}
$$


Aspect Ratio is calculated as the proportional difference between the graphs aspect ratio and the viewing panels aspect ratio.

$$
\begin{aligned}
& \text { Aspect }_{\text {Graph }}=\frac{\text { Width }_{\text {Graph }}}{\text { Height }_{\text {Graph }}} \text { Aspect }_{\text {View }}=\frac{\text { Width }_{\text {View }}}{\text { Height }_{\text {View }}} \\
& \text { Metric }_{\text {Aspect }}=\frac{\left.{\operatorname{Max}\left(\text { Aspect }_{\text {Graph }}, \text { Aspect }_{\text {View }}\right)}_{\text {Min } \left.\text { Aspect }_{\text {Graph }}, \text { Aspect }_{\text {View }}\right)}\right)}{-1}
\end{aligned}
$$

Edge Overlaps is an extension of Edge Crossings as it measures, for each pair of intersecting edges, the ratio of length of the edge compared to the length of the smallest line-segment created by splitting the edge at their point of intersection. Minimising this has the effect of trying to push the centres of the edges away from each other, minimising the distance the edges overlap by.

Nearest Neighbour Distance minimises the variance in distance between closest neighbours, trying to make the minimum distance between neighbouring nodes equal.

Uniform Edge Length minimises the variance in edge lengths, trying to make each edge the same length.

\subsection{Preliminary Results}

The graphs below were processed using a hill climbing multi-criteria optimising system. This system measures the quality of layout by finding the values for several metrics, weighting each metric and adding the weighted values to find an overall value for the quality of the layout. The graph is then modified in an attempt to reduce this overall value, and so improve the layout.

Our hill climber iterates through the nodes, testing each node by moving it to eight compass points. The node is moved to the point that has the lowest overall value for the metrics, or if the original position is lowest, no move is made. The hill climber starts by making 10 pixel moves until it completes a full iteration in which no node has moved. At this point it decrements the movement value and continues the process to find a solution with a better resolution until the movement value reduces to zero when the iterations stop. This approach was chosen to allow rapid movement towards the optimal solution and then fine tuning of the solution.

\begin{tabular}{l|l} 
Metric & Weighting \\
\hline Angular Resolution & 0.01 \\
Aspect Ratio & 0.000001 \\
Edge Overlaps & 1.0 \\
Nearest Neighbour Distance & 0.0001 \\
Node-Edge Distance & 0.01 \\
Uniform Edge Length & 0.001 \\
\hline Concentration & 1.0 \\
Homogeneity & 1.0
\end{tabular}

The graphs use six current metrics. Each was given a weighting, shown in the table above. These values were decided by the investigators, based on an attempt to both normalise the metrics, so that the resultant values are in the same order of magnitude. In addition, the two new metrics are given a high relative weighting to emphasise their impact on the results.

The following graphs are produced using the same starting graphs and identical weightings for the six traditional metrics.

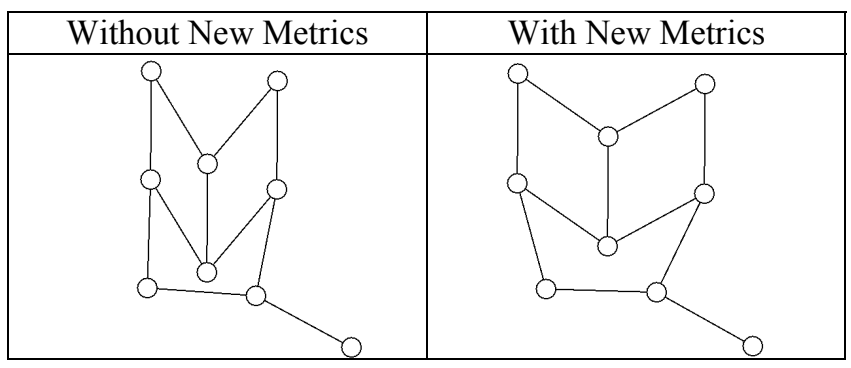

\begin{tabular}{l|l|l|l} 
Metric & Value & Weight & Total \\
\hline Angular Res. & 0.0 & 0.01 & 0.0 \\
Aspect Ratio & 0.478113 & 0.0000001 & $4.78 \times 10-7$ \\
Edge Overlaps & 0.0 & 1.0 & 0.0 \\
Nearest N'bour & 35.68553 & 0.00001 & $3.57 \times 10-3$ \\
N-E Distance & 0.0 & 0.01 & 0.0 \\
Uniform Edges & 0.119961 & 0.001 & $1.20 \times 10-4$ \\
\hline Concentration & 0.25 & 1.0 & 0.25 \\
Homogeneity & 0.666667 & 1.0 & 0.666667 \\
\hline \multicolumn{3}{|c|}{ Overall layout quality } & 0.920356
\end{tabular}

As an illustration of the actual values of the metrics, above are the values for the metrics for the graph with the hill climber not including the graphical design metrics and below are the results when the design metrics are included. The values for graphical design metrics are included in both tables.

\begin{tabular}{l|l|l|l} 
Metric & Value & Weight & Total \\
\hline Angular Res. & 0.0 & 0.01 & 0.0 \\
Aspect Ratio & 0.271946 & 0.0000001 & $2.72 \times 10-7$ \\
Edge Overlaps & 0.0 & 1.0 & 0.0 \\
Nearest N'bour & 27.69453 & 0.00001 & $2.77 \times 10-3$ \\
N-E Distance & 0.0 & 0.01 & 0.0 \\
Uniform Edges & 0.154999 & 0.001 & $1.55 \times 10-4$ \\
\hline Concentration & 0.0 & 1.0 & 0.0 \\
Homogeneity & 0.666667 & 1.0 & 0.666667 \\
\hline
\end{tabular}

\begin{tabular}{l|l} 
Overall layout quality & 0.669591
\end{tabular}

The results show that concentration metric has improved significantly using the graphical design metrics and this can be seen in the graph by the improved spread of nodes over the canvas. This is also reflected in the nearest neighbour separation and shows an almost $25 \%$ reduction in the variance in distance between the closest nodes. There has, however, been an increase in the variance of the uniformity of edge lengths but the numerical results show this is not as significant as the 
nearest neighbour reductions and does not show upon visual inspection of the graphs.

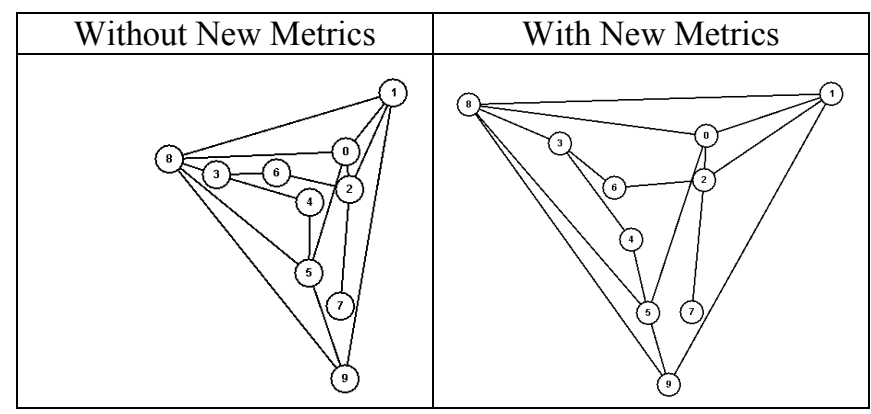

The graphs above show improvements in both concentration and homogeneity when using the new metrics. The graph produced using only the existing metrics has no nodes in the lower left quadrant. The graphical design based metrics change this, moving two nodes over the border between quadrants giving optimal values for both new metrics.

Visual comparison of the graphs show the new metrics have produced a greater node-edge resolution and the graph does not look as cluttered.

Without New Metrics

The graphical design metrics offer a subtle improvement over the existing metrics. Increasing the area of the graph, spreading the nodes out and generally increasing the node-edge separation and angular resolution. This gives the apparent result of making the graph less cluttered.

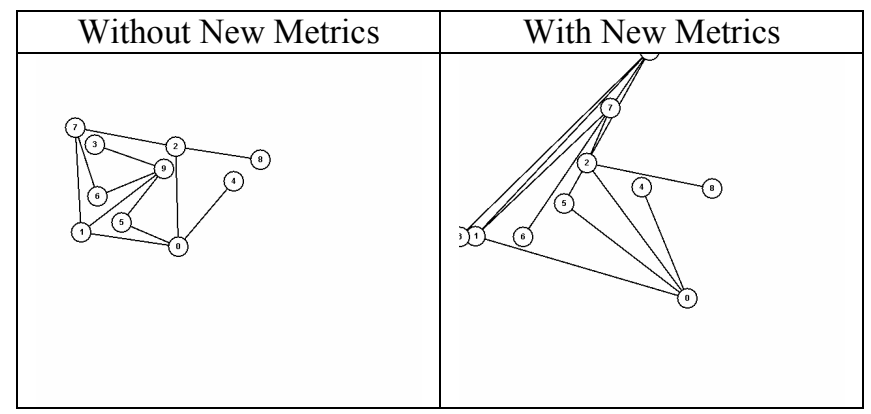

The layout produced using the new metrics shows a poor result in this case. By trying to improve the spread of the nodes the angular resolution and node-edge separation has been sacrificed. This can be improved by increasing the weighting of these metrics.

\section{Conclusions and Further Work}

We have described our preliminary work in applying graphic design criteria to graph visualization. The criteria make subtle alterations to the layout, which should have a beneficial effect on users' comprehension of data represented as a graph.

A major focus of our future investigation will be the implementation of improvements to the current metrics and the introduction of further metrics. Criteria describing the effective use of white space, regularity and unity are all possible criteria for which metrics could be developed.

Future work will also look at application areas for graph visualization. This paper has described the effect of the new metrics on abstract graphs. However, to fully apply the benefit of design techniques, the metrics must be applied in the context of an application. This may also result in new criteria that are application dependent.

\section{References}

[1] ARTS 350 Course Euphrates, William Paterson University http://euphrates.wpunj.edu/courses/arts350 /aesthetics.html

[2] Giuseppe Di Battista, Peter Eades, Roberto Tamassia, Ioannis G. Tollis. Graph Drawing, Algorithms for the Visualisation of Graphs. Prentice Hall 1999 - ISBN 013-301615-3

[3] G. Bonsiepe A Method of Quantifying Order in Typographic Design Journal of Typographic Research 2 (1968) 203-220.

[4] Michael Kaufmann, Dorothea Wagner (Eds). Drawing Graphs: Methods and Models. Springer Publishers 1998 - ISBN 3-540-42062-2

[5] A. Marcus. Graphic Design for Electronic Documents and User Interfaces ACM Press, New York, 1992.

[6] David Chek Ling Ngo, Lian Seng Teo and John G. Byrne. Modelling Interface Aesthetics. In Information Sciences 152 (2003) 25-46. Summarised at http://www.mi.sanu.ac.yu/vismath/ngo/

[7] Helen C. Purchase, Robert F. Cohen, Murray James. Validating Graph Drawing Aesthetics. In F. J. Brandenburg, editor, Graph Drawing (Proc. GD '95), vol. 1027 of Lecture Note in Computer Science, pp. 435446. Springer-Verlag, New York, 1985.

[8] N. Tractinsky, Aesthetics and Apparent Usability: Empirically Assessing Cultural and Methodological Issues. In CHI '97 Conference Proceedings, Association for Computing Machinery, New York, 1997

[9] T. S. Tullis An Evaluation of Alphanumeric, Graphic, and Colour Information Displays Human Factors 23 (1981) 541-550. 